\title{
Photoimmunotherapy Targeting Prostate-Specific Membrane Antigen: Are Antibody Fragments as Effective as Antibodies?
}

\author{
Rira Watanabe ${ }^{1}$, Hirofumi Hanaoka ${ }^{1}$, Kazuhide Sato ${ }^{1}$, Tadanobu Nagaya ${ }^{1}$, Toshiko Harada ${ }^{1}$, Makoto Mitsunaga ${ }^{1}$, \\ Insook Kim ${ }^{2}$, Chang H. Paik ${ }^{3}$, Anna M. Wu ${ }^{4}$, Peter L. Choyke ${ }^{1}$, and Hisataka Kobayashi ${ }^{1}$ \\ ${ }^{I}$ Molecular Imaging Program, Center for Cancer Research, National Cancer Institute, National Institutes of Health, Bethesda, \\ Maryland; ${ }^{2}$ Applied/Developmental Research Directorate, Leidos Biomedical Research Inc., Frederick National Laboratory, \\ Frederick, Maryland; ${ }^{3}$ Nuclear Medicine Department, Radiology and Imaging Science, Warren Grant Magnuson Clinical Center, \\ National Institutes of Health, Bethesda, Maryland; and ${ }^{4}$ Department of Molecular and Medical Pharmacology, David Geffen School \\ of Medicine at UCLA, Los Angeles, California
}

Photoimmunotherapy is a highly cell-selective cancer therapy based on an armed antibody conjugate with a phthalocyaninebased photosensitizer, IR700. Photoimmunotherapy induces rapid and highly specific necrosis in targeted cancer cells after exposure to near-infrared (NIR) light. Cells not expressing the antigen are not affected. To date, photoimmunotherapy has been demonstrated only with full antibody-IR700 conjugates. In this study, small and bivalent antibody fragments, including anti-prostate-specific membrane antigen (PSMA) diabody (Db) and minibody (Mb), were compared with intact IgG for their effectiveness as photoimmunotherapy agents. Methods: Radioiodinated antibody and antibody fragments with ${ }^{125}$ I were used to determine the timing of maximum binding of each anti-PSMA antibody fragment on the cell surface in vivo in mice bearing either PSMA-positive or -negative PC3 tumors. Then therapeutic efficacy of photoimmunotherapy was examined by exposing mice to NIR light at 2 time points based on the time of maximum cell surface binding at $6 \mathrm{~h}$ after injection for Db-IR700 and $24 \mathrm{~h}$ after injection for Mb-IR700 and IgG-IR700 as well as $24 \mathrm{~h}$ after the peak uptake times. Results: Photoimmunotherapy with the same molar concentration of PSMA-Db-IR700, PSMA-Mb-IR700, and PSMA-IgG-IR700 conjugate showed similar therapeutic effects in vitro and in vivo on PSMA-positive PC3 tumor xenografts in cytotoxicity and survival curves $(P>0.05)$. Conclusion: The use of PSMA-Db-IR700 conjugate results in the shortest time interval between injection and NIR exposure without compromising therapeutic effects of photoimmunotherapy.

Key Words: photoimmunotherapy; prostate specific membrane antigen; monoclonal antibody; diabody; minibody; pharmacokinetics

J Nucl Med 2015; 56:140-144

DOI: 10.2967/jnumed.114.149526

\section{A}

ntibody-based cancer therapies, including therapeutic monoclonal antibodies, antibody-drug conjugates, antibody-toxin conjugates (immunotoxin), and radioimmunoconjugates, have been

\footnotetext{
Received Oct. 3, 2014; revision accepted Nov. 7, 2014.

For correspondence or reprints contact: Hisataka Kobayashi, Molecular Imaging Program, Center for Cancer Research, National Cancer Institute, National Institutes of Health, Building 10, Rm. B3B69, MSC1088, Bethesda, MD 20892-1088.

E-mail: Kobayash@mail.nih.gov

Published online Dec. 11, 2014.

COPYRIGHT (C 2015 by the Society of Nuclear Medicine and Molecular Imaging, Inc.
}

a mainstay of molecularly targeted therapy (1). The antibodies used in these conjugates typically demonstrate great affinity for the target; however, there are some well-known limitations to the use of whole antibodies. Therapeutic antibodies do not initially permeate across the cell membrane, and thus they induce cytotoxicity at the cell surface, typically invoking antibody-dependent cellular cytotoxicity or complement-dependent cytotoxicity (1). Antibody-drug conjugates or immunotoxins can release their therapeutic payload after binding and internalization (2,3). Radioimmunoconjugates carry therapeutic radioisotopes that lead to radiationinduced cell death without internalization (4). These approaches have been successful in treating cancers; however, hepatic, renal, and bone marrow exposure often results in dose-limiting toxicities.

Photoimmunotherapy represents yet another way of harnessing antibodies to direct therapy. In photoimmunotherapy, an antibody is conjugated to a phthalocyanine-based photosensitizer, IR700, and when the conjugate binds the target cell and is exposed to near-infrared (NIR) light, it results in rapid and highly specific target cell death (5). Unlike other immunoconjugates, photoimmunotherapy can limit off-target side effects by 2 safety check points: it requires the conjugate to be docked at the cell surface and it requires the presence of light to activate. Because the conjugate is only effective after binding to target molecules on the cellular membrane, there are few off-target side effects and further safety can be achieved by limiting the region of exposure to NIR light. However, full antibodies, because of their relatively large size, do not penetrate evenly into tumor parenchyma, limiting the extent of therapy. Thus, genetically engineered small and bivalent antibody fragments, which are considerably smaller, might also bind more quickly and stably to the antigen yet improve the intratumoral distribution of the conjugate (6). Therefore, in this study, a small and bivalent anti-prostate-specific membrane antigen (PSMA) diabody $(\mathrm{Db})$ and minibody $(\mathrm{Mb})$ conjugated to IR700 were compared with an IgG-IR700 conjugate in a PSMA-positive tumor model. The timing of maximum binding to PSMA on the cell surface was determined for each antibody type, and therapeutic efficacy of photoimmunotherapy with the 3 agents was compared.

\section{MATERIALS AND METHODS}

\section{Reagents}

HuJ591, a humanized PSMA-specific monoclonal antibody (IgG), was developed at Weill Cornell Medical College and kindly provided by Prof. Neil H. Bander. Anti-PSMA-Mb and anti-PSMA-cys-Db were kindly supplied by ImaginAb Inc. IRdye 700-DX (IR700) NHS 
ester was purchased from LI-COR Biosciences. All other chemicals were of reagent grade.

\section{Synthesis of IR700-Conjugated IgG, Mb, and Db}

$\operatorname{IgG}(1.0 \mathrm{mg}, 6.2 \mathrm{nmol}), \mathrm{Mb}(0.5 \mathrm{mg}, 6.3 \mathrm{nmol})$, or $\mathrm{Db}(0.5 \mathrm{mg}, 10$ nmol) was incubated with IR700 (molar ratio, 1:5) in $0.1 \mathrm{M} \mathrm{Na}_{2} \mathrm{HPO}_{4}$ ( $\mathrm{pH}$ 8.6) at room temperature for $30 \mathrm{~min}$, followed by purification with a size-exclusion Sephadex G-25 M column (PD-10; GE Healthcare). The concentration of IR700 was calculated by measuring the absorption with a UV-Visible Value System (model 8453; Agilent Technologies) to confirm the number of fluorophore molecules conjugated to IgG, Mb, or Db. The number of IR700 per IgG, Mb, or Db was 2-4 (7).

\section{Radioiodination of IgG, Mb, and $\mathrm{Db}$}

${ }^{125}$ I-PSMA-IgG, -Mb, and -Db were prepared using the IODOGEN (Pierce) procedure. Briefly, $100 \mu \mathrm{g}$ of each protein (PSMA$\mathrm{IgG}, \mathrm{Mb}$, or $\mathrm{Db}$ ) were added to each IODO-GEN-coated vial and iodinated with $37 \mathrm{MBq}$ of ${ }^{125} \mathrm{I}-\mathrm{Na}$, which was neutralized to $\mathrm{pH} 7.2$ with the addition of $0.5 \mathrm{M}$ phosphate buffer at room temperature. After $5 \mathrm{~min}$ of reaction, each ${ }^{125}$ I-radiolabeled product was purified with a PD-10 size-exclusion column. The specific activities of the radiolabeled $\mathrm{IgG}, \mathrm{Mb}$, and $\mathrm{Db}$ were $207.2 \mathrm{MBq}(5.6 \mathrm{mCi}) / \mathrm{mg}$ for PSMA-IgG, $251.6 \mathrm{MBq}(6.8 \mathrm{mCi}) / \mathrm{mg}$ for PSMA-Mb, and 347.8 $(9.4 \mathrm{mCi}) / \mathrm{mg}$ for PSMA-Db. The radiochemical purity of radiolabeled products was validated with size-exclusion high-performance liquid chromatography equipped with a TSK SWxl G3000 column (TosoHaas), an ultraviolet monitor, and an online radiodetector (Bioscan). The high-performance liquid chromatography was eluted with $0.067 \mathrm{M}$ phosphate-buffered saline (PBS) with $100 \mathrm{mM} \mathrm{KCl}$ at a flow rate of $1 \mathrm{~mL} / \mathrm{min}$.

\section{Cell Culture}

A PSMA-transfected PC3 cell line, PSMA-positive (PC3pip) cells, and a control blank vector transfected PC 3 cell line, PSMA-negative (PC3flu) cells, were used for PSMA-targeting studies. Both cells were established at the Cleveland Clinic Foundation and kindly provided by Prof. Warren Heston. All cell lines were grown in RPMI 1640 medium (Life Technologies) containing 10\% fetal bovine serum (Life Technologies) and 1\% Pen-Strep (Life Technologies). All cell cultures were maintained in $5 \%$ carbon dioxide at $37^{\circ} \mathrm{C}$ in a humidified incubator.

\section{Fluorescence Microscopy Studies}

PSMA-positive or -negative cells $\left(1 \times 10^{4}\right)$ were plated on a covered glass-bottomed culture well and incubated overnight. Then IgG-IR700, Mb-IR700, or Db-IR700 was added to the medium at $10 \mu \mathrm{g} / \mathrm{mL}$, and the cells were incubated for $5 \mathrm{~h}$ at $37^{\circ} \mathrm{C}$ in a humidified incubator or for $1 \mathrm{~h}$ on ice. Cells were washed once with PBS, and fluorescence microscopy was performed using an Olympus BX61 microscope (Olympus America, Inc.) equipped with the following filters: excitation wavelength, 590-650 nm; emission wavelength, 665-740 nm. Transmitted light differential interference contrast images were also acquired before and after the fluorescence imaging.

\section{In Vitro Photoimmunotherapy}

Cells were seeded into 35-mm cell culture dishes and incubated overnight. Medium was replaced with phenol red-free culture medium containing IgG-IR700 (10 $\mu \mathrm{g} / \mathrm{mL})$, Mb-IR700 (5 $\mu \mathrm{g} / \mathrm{mL})$, or DbIR700 $(3 \mu \mathrm{g} / \mathrm{mL})$ that was the same molar concentration at $70 \mathrm{nM}$. After incubation for $5 \mathrm{~h}$ at $37^{\circ} \mathrm{C}$, phenol red-free culture medium was added. Then cells were irradiated with a red light-emitting diode, which emits light at a 670- to 710-nm wavelength (L690-66-60; Marubeni America Co.), and a power density of $25 \mathrm{~mW} / \mathrm{cm}^{2}$ as measured with optical power meter (PM 100; Thorlabs). All in vitro experiments were repeated at least 3 times.

\section{Phototoxicity Assay}

Cytotoxic effects of photoimmunotherapy with IgG-IR700, Mb-IR700, or Db-IR700 were determined with a flow cytometric LIVE/DEAD Fixable Green Dead Cell Stain Kit (Life Technologies), which can detect compromised cell membranes. Cells were trypsinized after treatment and washed with PBS. Green fluorescent reactive dye was added in the cell suspension and incubated at room temperature for $30 \mathrm{~min}$, followed by analysis on a flow cytometer (FACS Calibur; BD Biosciences).

\section{Animal Tumor Model}

All procedures were performed in compliance with the Guide for the Care and Use of Laboratory Animals (8), National Research Council, and approved by the local Animal Care and Use Committee. Six- to 8-wk-old female homozygote athymic nude mice were purchased from Charles River (NCI-Frederick). During the procedure, mice were anesthetized with isoflurane. PSMA-positive or -negative cells $\left(2 \times 10^{6} /\right.$ mouse, suspended in PBS $)$ were injected subcutaneously in the dorsum of the mice.

\section{Biodistribution Study}

PSMA-positive and -negative tumor-bearing mice were divided into $3-4$ groups $(n=3-5)$ with approximately equal distributions of tumor sizes on the day of study, $5 \mathrm{~d}$ after inoculation of the cells. ${ }^{125} \mathrm{I}-\mathrm{IgG}$, $-\mathrm{Mb}$, or $-\mathrm{Db}(37 \mathrm{kBq} / 1.0 \mu \mathrm{g} / 200 \mu \mathrm{L}$ in PBS per mouse) was injected via the tail vein. The biodistribution of ${ }^{125} \mathrm{I}-\mathrm{IgG},-\mathrm{Mb}$, or $-\mathrm{Db}$ was determined at $6 \mathrm{~h}$ and 1,2 , and $3 \mathrm{~d}$ after injection; at 1 and $6 \mathrm{~h}$ and 1 and $3 \mathrm{~d}$ after injection; or at 1,6 , and $24 \mathrm{~h}$ after injection, respectively, based on their reported biologic clearance rate. Organs of interest were excised and weighed, and the radioactivity counts were determined with a $\gamma$ counter (Wizard 2480; PerkinElmer) using the injected dose as a standard. Data were calculated as the percentage injected dose per gram of tissue.

\section{In Vivo Photoimmunotherapy}

PSMA-positive tumor-bearing mice were allocated on the basis of the tumor volume $3 \mathrm{~d}$ after inoculation of the cells to each of 3 groups of 9-10 animals per group for the following treatments: no treatment control; the same mole dose (670 pmol) of agent (100 $\mu \mathrm{g}$ of IgG-IR700, $50 \mu \mathrm{g}$ of Mb-IR700, or $30 \mu \mathrm{g}$ of Db-IR700) intravenously (27 pmol/kg), no NIR light exposure; and the same mole dose of agent intravenously, NIR light administered at $50 \mathrm{~J} / \mathrm{cm}^{2}$ on day 1 and $100 \mathrm{~J} / \mathrm{cm}^{2}$ on day 2 after injection of IgG-IR700 and Mb-IR700, or at $50 \mathrm{~J} / \mathrm{cm}^{2}$ at $6 \mathrm{~h}$ and $100 \mathrm{~J} / \mathrm{cm}^{2}$ on day 1 after injection of Db-IR700. To determine tumor volume, the greatest longitudinal diameter (length) and the greatest transverse diameter (width) were measured with an external caliper. Tumor volumes based on caliper measurements were calculated using tumor volume $=$ length $\times(\text { width })^{2} \times 0.5$. Mice were observed daily, and tumor volumes were measured 3 times a week until the tumor volume had reached more than $500 \mathrm{~mm}^{3}$, at which time mice had to be euthanized with carbon dioxide.

\section{Statistical Analysis}

Quantitative data were expressed as mean \pm SEM from a minimum of 3 experiments. The Dunnett test was used for multiple comparisons of tumor volumes. The cumulative probability of survival was estimated in each group with the Kaplan-Meier survival curve analysis, and the results were compared using the log-rank test. A $P$ value of less than 0.05 was considered statistically significant.

\section{RESULTS}

Target-Specific, Dose-Dependent NIR Light-Induced Necrotic Cell Death in Response to Photoimmunotherapy

Fluorescence microscopy was performed to confirm target-specific localization of each conjugate. IR700 was mainly localized to the cell 
membrane after $1 \mathrm{~h}$ of coincubation on ice and to the lysosomes of PSMA-positive cells at room temperature. When these cells were observed during continuous NIR light exposure, almost immediate swelling, budding, and rupture of the lysosome were observed, leading to irreversible cell death (Fig. 1). PSMA-positive and PSMA-negative cells were coincubated to confirm cell-specific killing. IgG-IR700, Mb-IR700, and Db-IR700 did not localize to PSMA-negative cells, which were not killed by exposure to NIR light (Fig. 1).

To confirm that rapid phototoxic cell death had occurred, we used the LIVE/DEAD assay (Life Technologies), which can detect early cell membrane damage. Death of PSMA-positive cells by photoimmunotherapy with IgG-IR700, Mb-IR700, or Db-IR700

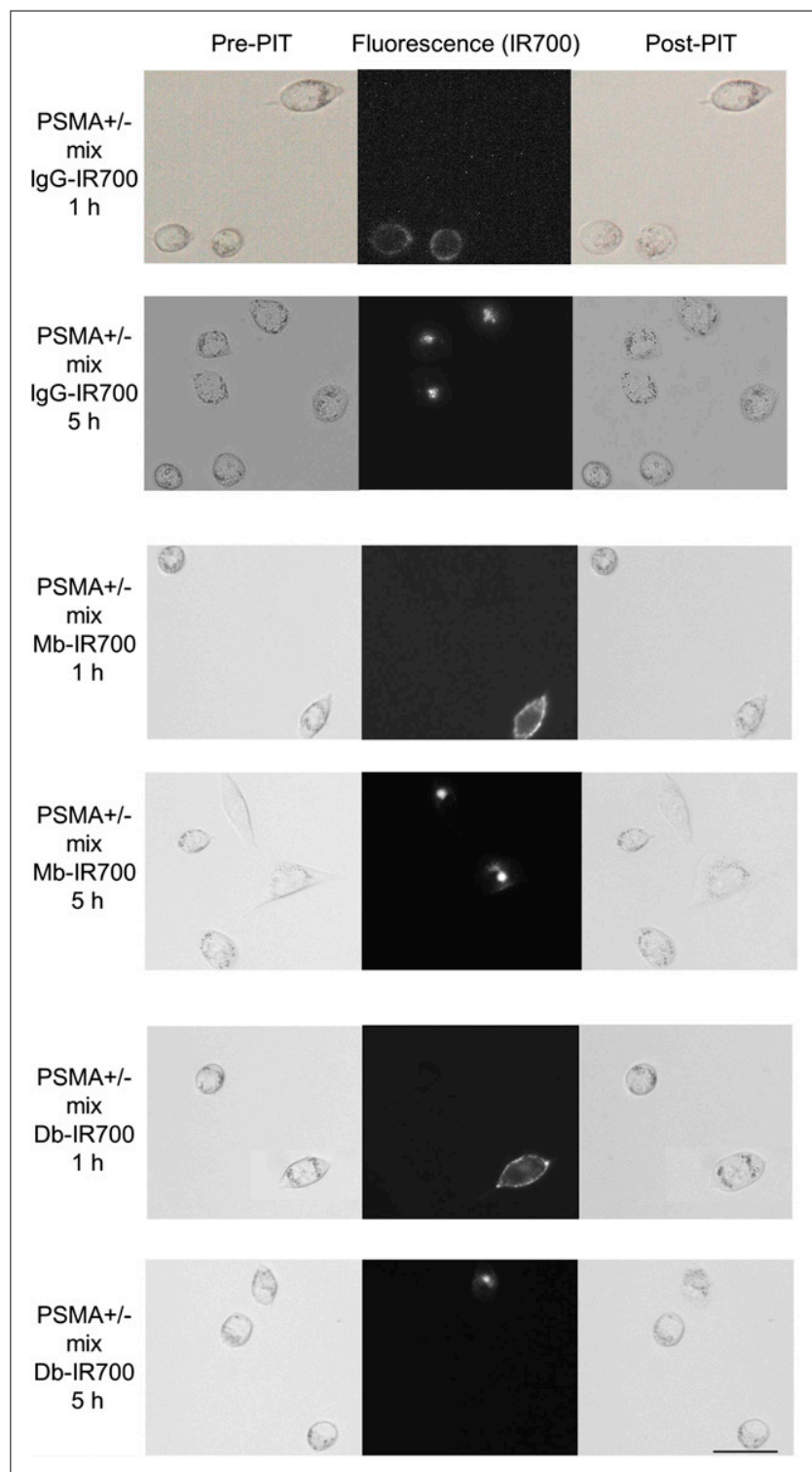

FIGURE 1. Differential interference contrast and fluorescence microscopy of photoimmunotherapy-treated mix of PC3pip (PSMA-positive) and flu (PSMA-negative) cells. Cells were incubated with anti-PSMA antibodies (IgG-IR700), Mbs (Mb-IR700), or Dbs (Db-IR700) at $10 \mu \mathrm{g} / \mathrm{mL}$. Just after fluorescence imaging, only PSMA-positive cells demonstrate necrotic cell death (cell budding/rupture). Scale bar $=50 \mu \mathrm{m}$. PIT $=$ photoimmunotherapy. increased in a dose-dependent manner with the intensity of NIR light. No cell death was observed in the absence of IgG-IR700 or Mb-IR700 or in the absence of light, whereas considerable cell death was observed with either unlabeled $\mathrm{Db}$ or Db-IR700 without NIR irradiation (Fig. 2A). Cell death by photoimmunotherapy with those IR700 conjugates or unlabeled agents was not observed in PSMA-negative cells (Fig. 2B).

\section{Biodistribution of ${ }^{125} \mathrm{I}-\mathrm{IgG}$, $-\mathrm{Mb}$, or -Db}

Results of in vivo biodistribution studies of ${ }^{125} \mathrm{I}-\mathrm{IgG},{ }^{125} \mathrm{I}-\mathrm{Mb}$, and ${ }^{125} \mathrm{I}-\mathrm{Db}$ were expressed as percentage injected dose per gram (Supplemental Fig. 1 [supplemental materials are available at http://jnm.snmjournals.org]) and as ratios to blood (Fig. 3, left), as a subtraction of PSMA-negative tumor values from PSMApositive tumor values (Fig. 3, center), and as ratios of PSMAnegative tumor to PSMA-positive tumor (Fig. 3, right). The highest activity was observed in the spleen, kidney, and PSMA-positive tumors. Compared with IgG, Db distributed to the kidney immediately after injection. High radioactivity in the stomach at $6 \mathrm{~h}$ after injection suggests rapid catabolism. Clearance of $\mathrm{Mb}$ was also much faster than IgG; however, there was no evidence of rapid catabolism.

The peak difference of percentage injected dose per gram between PSMA-positive and -negative tumors was at $1 \mathrm{~d}$ after injection for IgG and $\mathrm{Mb}$ but was $6 \mathrm{~h}$ for $\mathrm{Db}$ (Fig. 3, center).

\section{Tumor Growth Inhibition in Response to Photoimmunotherapy}

PSMA-positive tumors were treated with photoimmunotherapy using 2 exposures to NIR light at $50 \mathrm{~J} / \mathrm{cm}^{2}$ on the peak time of tumor uptake as shown by biodistribution and at $100 \mathrm{~J} / \mathrm{cm}^{2} 1 \mathrm{~d}$ later. In the case of IgG-IR700 or Mb-IR700, the first light exposure took place at $24 \mathrm{~h}$ and the second at $48 \mathrm{~h}$ after injection whereas for Db-IR700 the first light exposure took place at $6 \mathrm{~h}$ and the second at $24 \mathrm{~h}$ after injection.

Tumor growth was significantly inhibited in the photoimmunotherapy treatment groups with IgG-IR700, Mb-IR700, or DbIR700, and significantly prolonged survival was observed in photoimmunotherapy treatment groups with IgG-IR700 or MbIR700 (Fig. 4). No significant therapeutic effect was observed in the group of each agent only, without NIR irradiation (Fig. 4), or in mice with NIR irradiation only, without injection of agent (Supplemental Fig. 2). No significant difference on survival was detected between different agents $(P>0.05)$.

\section{DISCUSSION}

Photoimmunotherapy is a highly selective method of killing cancer cells that depends on exposure to NIR light. Typically a full $\mathrm{IgG}$ monoclonal antibody is conjugated with the phthalocyanine dye IR700. Approximately $24 \mathrm{~h}$ after the injection, when the immunoconjugate is bound to the appropriate cell surface targets, exposure to NIR activates the photosensitizer and selectively kills the cells to which the antibody is bound (5,9-11). Although whole IgGs have the advantage of high input function due to prolonged clearance time from the circulation, antibody fragments such as Dbs and Mbs have theoretic advantages based on their better penetration into tumor, resulting in homogeneous microdistribution. Therefore, this study was conducted to compare the efficacy of photoimmunotherapy using a full antibody and antibody fragments. To determine the optimal binding kinetics of antibody fragments to the PSMA expressed on the cell membrane, we used ${ }^{125}$ I-labeled 


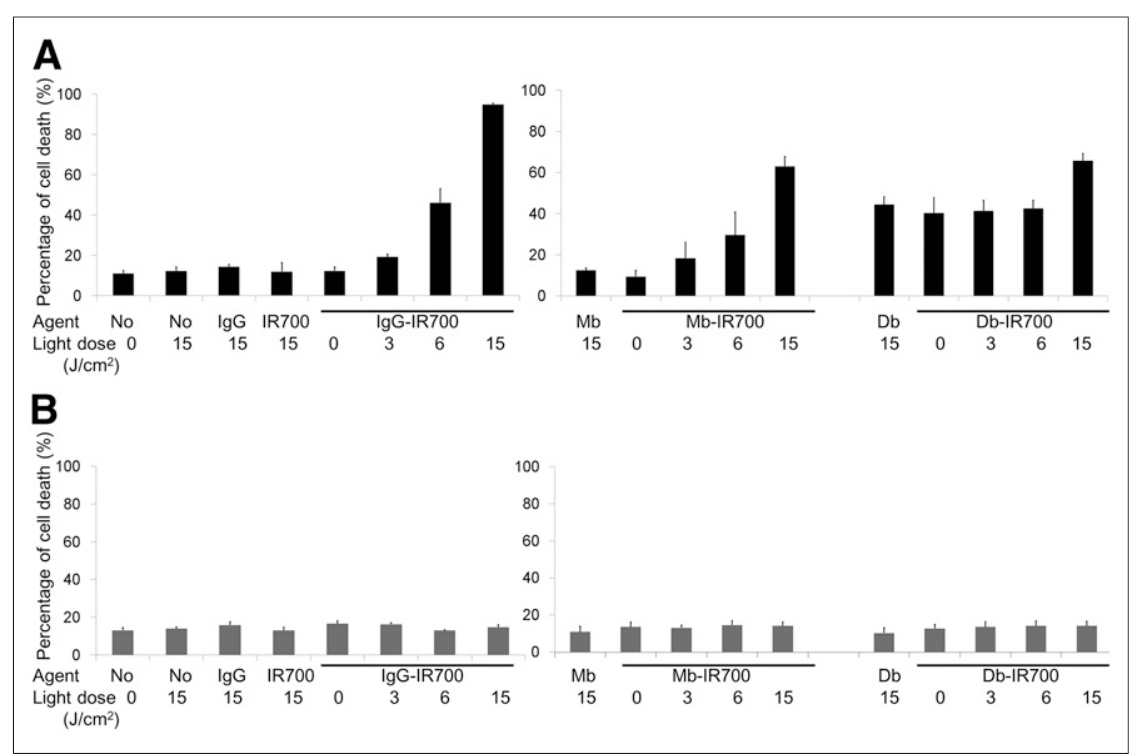

FIGURE 2. Target-specific cell death in response to IgG-, Mb-, or Db-IR700-mediated photoimmunotherapy in PSMA-positive (A) or -negative (B) cells. Target-specific cell death in response to photoimmunotherapy was dose-dependent to intensity of irradiation. Data are mean \pm $\operatorname{SEM}(n \geq 3)$.

antibody fragments in mice bearing both PSMA-positive and PSMA-negative PC3 tumors. Because radioiodine detaches from the cell when radiolabeled fragments are internalized, we could detect the surface-bound fraction of antibody fragments by comparing

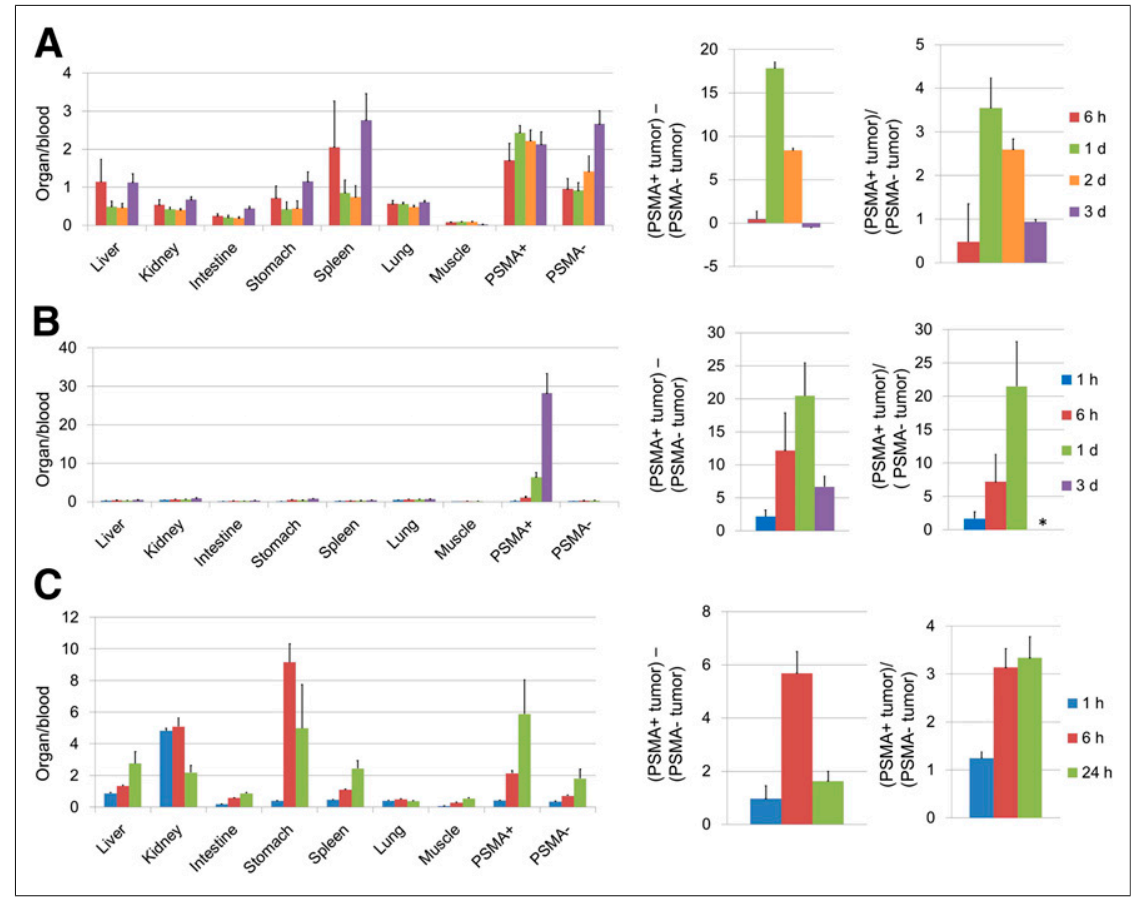

FIGURE 3. Analysis of in vivo biodistribution of radioactivity after injection of ${ }^{125}$-PSMA-IgG (A), $\mathrm{Mb}(\mathrm{B})$, or $\mathrm{Db}(\mathrm{C})$ into mice bearing PSMA positive (PSMA+) and negative (PSMA-) tumors. Each value was calculated using percentage injected dose per gram of tissue (Supplemental Fig. 1) for each animal and represented as mean \pm SEM $(n=3-5)$ : ratios of each organ and tumor to blood (left), subtraction of PSMA-negative tumor from PSMA-positive tumor (center), and ratios of PSMA-positive tumor to PSMA-negative tumor (right). * = value cannot be calculated because some denominators are 0.
PSMA-positive tumor with PSMA-negative controls (12). Although PSMA is internalized rapidly, IR700 bound to cell membrane and photoimmunotherapy effects were similarly confirmed at both 1 and $5 \mathrm{~h}$ after coincubation with agents regardless of different internalized amounts of IR700. In addition, the biodistribution data shown in Figure 3 indicated that the optimal binding time for PSMA-targeted molecules based on $\mathrm{Db}, \mathrm{Mb}$, and $\mathrm{IgG}$ was at 6,24 , and $24 \mathrm{~h}$ after injection, respectively. Therefore, for Db-IR700 conjugates NIR exposure in PSMA-positive PC3 tumor-bearing mice was performed at 6 and $24 \mathrm{~h}$, whereas Mb-IR700 and IgG-IR700 conjugates required NIR exposure at 24 and $48 \mathrm{~h}$ after injection in the same model. Although all conjugates produced comparable cell killing with photoimmunotherapy, the early treatments with Db-IR700 is a comparative advantage for this antibody fragment. Despite different accumulation doses of the 3 conjugates, growth suppression effects induced by photoimmunotherapy were similar in all 3 groups. The higher input function due to longer clearance time of the larger fragments was likely balanced by the improved microdistribution of the smaller fragments. This 2-time irradiation regimen is considered to improve the efficacy of agent delivery into the tumor, because the first treatment kills tumor cells especially near the vessels, inducing a post-photoimmunotherapy superenhanced permeability and retention effect, which enhances delivery of any macromolecules subsequently administered into the tumor bed $(9,11)$. Therefore, we assessed biodistribution of all agents and adopted the first time point as the peak tumor uptake of ${ }^{125} \mathrm{I}$ and the second as $1 \mathrm{~d}$ later when macromolecular intratumoral microdistribution was optimal because of the photoimmunotherapy-induced superenhanced permeability and retention effect (11). The treatment regimen and irradiation timing for $\mathrm{Mb}$ and $\mathrm{Db}$ might be further optimized in the clinical setting because the clearance is different in humans, compared with mice.

As shown in Figure 1, target-specific necrotic cell death was similar in all 3 groups at 1 and $5 \mathrm{~h}$ after incubation. The DbIR700 conjugate showed greater selective cytotoxicity to PSMA-positive cells than PSMA-negative cells even without exposure of NIR light at $5 \mathrm{~h}$ after incubation as shown in Figure 2. However, in vivo cytotoxicity of Db-IR700 conjugate by itself was not observed, and cell killing was seen only with NIR exposure. We assessed cytotoxicity of Db and Db-IR700 and found that both had dose-related cytotoxicity and the 


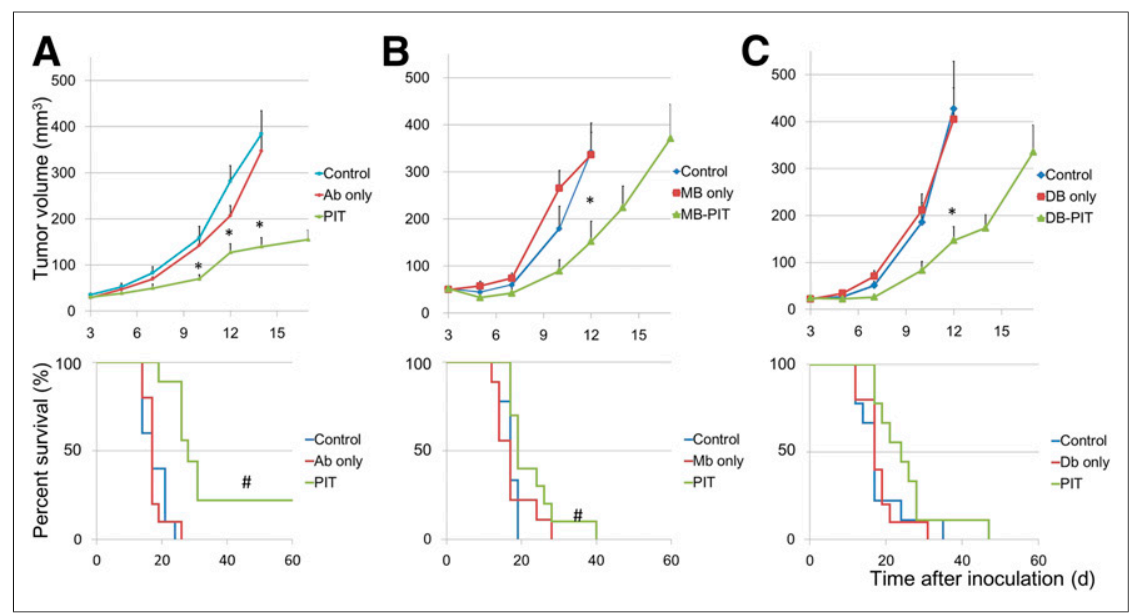

FIGURE 4. Tumor volume and survival curves of PSMA-positive tumor-bearing mice. Mice were randomized to 1 of 3 groups at $3 \mathrm{~d}$ after tumor cell injection $(n=9-10)$. IgG-IR700 (A), Mb-IR700 (B), or Db-IR700 (C) was injected, and photoimmunotherapy was performed at 1 and $2 \mathrm{~d}$ after injection for IgG-IR700 or Mb-IR700 and at $6 \mathrm{~h}$ and $1 \mathrm{~d}$ after injection of Db-IR700. Mice were observed until tumor volume reached more than $500 \mathrm{~mm}^{3}$, at which time mice had to be euthanized. ${ }^{*} P<0.05$ vs. control, Dunnett multiple-comparison test. $\# P<0.05$ vs. control, log-rank test. $A b=$ antibody. the Intramural Research Program of the National Institutes of Health, National Cancer Institute, Center for Cancer Research. This project has been funded in whole or in part with federal funds from the National Cancer Institute, National Institutes of Health, under Contract No. HHSN261200800001E. The content of this publication does not necessarily reflect the views or policies of the Department of Health and Human Services, nor does mention of trade names, commercial products, or organizations imply endorsement by the U.S. Anna M. Wu is a shareholder and consultant to Imagin$\mathrm{Ab}$, Inc. The Regents of the University of California have licensed intellectual property to Imagin $\mathrm{Ab}$ and have taken equity as part of the licensing transaction. No other potential conflict of interest relevant to this article was reported.
LD50 (the lethal dose for 50\% [cell] death) was approximately 1-3 $\mu \mathrm{g} / \mathrm{mL}$ (data not shown), at which level $\mathrm{Db}$ saturated cell surface PSMA molecules. However, considering the rapid in vivo clearance of $\mathrm{Db}$, the actual concentration of Db-IR700 in tumor cells in vivo is likely to be far lower than this level. Therefore, all cytotoxicity that was shown in vivo required activation by NIR light exposure.

Inaccessibility of light to deep organs is a limitation of photoimmunotherapy even with NIR light; however, many tumors such as prostate cancer recurrences or regional lymph nodes would be amenable to photoimmunotherapy using laparoscopic probes. Although a light-emitting diode was used in this study, laser light also can be used and is more effective. Laser can be applied to endoscopy; therefore, photoimmunotherapy might be effective for treatment of disseminated tumor cells or tiny recurrences after surgery.

\section{CONCLUSION}

We demonstrate that equally effective photoimmunotherapy can be obtained with both full antibodies and antibody fragments. Because the pharmacokinetics of smaller fragments (e.g., Dbs) are faster, photoimmunotherapy can be initiated at a shorter time interval after smaller fragment injection than with larger fragments and intact antibodies, and this feature may have advantages in clinical translation of photoimmunotherapy. Therefore, the use of Db-IR700 conjugate can minimize the time interval between injection and NIR exposure without compromising the therapeutic effects of photoimmunotherapy despite inferior accumulation of the agent.

\section{DISCLOSURE}

The costs of publication of this article were defrayed in part by the payment of page charges. Therefore, and solely to indicate this fact, this article is hereby marked "advertisement" in accordance with 18 USC section 1734 . This research was supported by

\section{ACKNOWLEDGMENTS}

We thank Drs. Derek Bartlett, Christian P. Behrenbruch, and Jean Gudas at ImaginAb Inc. for their technical and administrative assistances.

\section{REFERENCES}

1. Waldmann TA, Morris JC. Development of antibodies and chimeric molecules for cancer immunotherapy. Adv Immunol. 2006;90:83-131.

2. Alley SC, Okeley NM, Senter PD. Antibody-drug conjugates: targeted drug delivery for cancer. Curr Opin Chem Biol. 2010;14:529-537.

3. Pastan I, Hassan R, Fitzgerald DJ, Kreitman RJ. Immunotoxin therapy of cancer. Nat Rev Cancer. 2006;6:559-565.

4. Sharkey RM, Goldenberg DM. Cancer radioimmunotherapy. Immunotherapy. 2011;3:349-370

5. Mitsunaga M, Ogawa M, Kosaka N, Rosenblum LT, Choyke PL, Kobayashi H. Cancer cell-selective in vivo near infrared photoimmunotherapy targeting specific membrane molecules. Nat Med. 2011;17:1685-1691.

6. Wu AM, Senter PD. Arming antibodies: prospects and challenges for immunoconjugates. Nat Biotechnol. 2005;23:1137-1146.

7. Sano K, Mitsunaga M, Nakajima T, Choyke PL, Kobayashi H. In vivo breast cancer characterization imaging using two monoclonal antibodies activatably labeled with near infrared fluorophores. Breast Cancer Res. 2012;14: R61.

8. Guide for the Care and Use of Laboratory Animals. Washington, DC: National Academy Press; 1996.

9. Mitsunaga M, Nakajima T, Sano K, Choyke PL, Kobayashi H. Near-infrared theranostic photoimmunotherapy (PIT): repeated exposure of light enhances the effect of immunoconjugate. Bioconjug Chem. 2012;23:604609.

10. Nakajima T, Sano K, Choyke PL, Kobayashi H. Improving the efficacy of Photoimmunotherapy (PIT) using a cocktail of antibody conjugates in a multiple antigen tumor model. Theranostics. 2013;3:357-365.

11. Sano K, Nakajima T, Choyke PL, Kobayashi H. Markedly enhanced permeability and retention effects induced by photo-immunotherapy of tumors. ACS Nano. 2013;7:717-724.

12. Kobayashi H, Kao CH, Kreitman RJ, et al. Pharmacokinetics of ${ }^{111}$ In- and ${ }^{125}$ I-labeled antiTac single-chain Fv recombinant immunotoxin. J Nucl Med. 2000;41:755-762. 